\title{
Importance analysis of a blog quality model for criteria and families in different blog categories
}

\begin{abstract}
A blog quality model has been proposed for bloggers to promote readers satisfaction. However, the model does not determine whether readers consider some quality criteria important, particularly with respect to different blog categories. In this paper, we employed a Rasch analysis to rank the importance of blog quality criteria for the Personal Diary, Sociopolitical Commentary, Humor/Entertainment, Lifestyle, and Technology blog categories. The authors identified the most important quality criteria and families for each category. The authors discovered that (1) the importance of quality criteria and/or families depends on the particular category with which a reader engages; (2) some quality criteria and/or families are more important for some categories but less important for others; and (3) certain quality criteria and/or families are equally important for some categories but not for others. The authors provide empirical evidence of the most important criteria bloggers and evaluators can focus on when they examine different blog categories. A blog quality model has been proposed for bloggers to promote readers satisfaction. However, the model does not determine whether readers consider some quality criteria important, particularly with respect to different blog categories. In this paper, a Rasch analysis to rank the importance of blog quality criteria for the Personal Diary, Socio-political Commentary, Humor/Entertainment, Lifestyle, and Technology blog categories. The authors identified the most important quality criteria and families for each category. The authors discovered that (1) the importance of quality criteria and/or families depends on the particular category with which a reader engages; (2) some quality criteria and/or families are more important for some categories but less important for others; and (3) certain quality criteria and/or families are equally important for some categories but not for others. The authors provide empirical evidence of the most important criteria bloggers and evaluators can focus on when they examine different blog categories.
\end{abstract}

Keyword: Blog quality; Blog quality model; Rasch analysis; Ranking; Weight 